\title{
Customer Centricity bei der Graubündner Kantonalbank - Kundenorientierung als Veränderungsprogramm
}

In reifen Branchen gleichen sich die Angebote zusehends an. Der Customer-Centricity-Ansatz bietet entscheidende Differenzierungschancen und Wettbewerbsvorteile. Doch wie schaffen es Unternehmen, eine Kultur zu entwickeln, in der eine kundenorientierte Arbeitsweise der Mitarbeiter zum Leitmotiv wird? Das Beispiel der Graubündner Kantonalbank zeigt, wie es gelingt, durch einen langfristigen und ganzheitlichen Veränderungsprozess das gesamte Unternehmen auf die Kundenschnittstelle auszurichten, Kunden zu begeistern und die Wertschöpfung nachhaltig zu erhöhen.

Alex Villiger, Dennis Herhausen, Marcus Schögel 
Die Schweizer Bankenbranche befindet sich in einem Strukturwandel. Das tiefe Zinsniveau, neue regulatorische Anforderungen sowie steigende Kosten stellen bewährte Geschäftsmodelle infrage. Zudem ist der Bankenmarkt gesättigt, verzeichnet geringe Wachstumsraten und ist von starker Konkurrenz geprägt. Den Banken stehen gut informierte Kunden gegenüber, die Produkte und Dienstleistungen kritisch prüfen und bezüglich Service, Kompetenz und Preis hohe Anforderungen stellen. In diesem verschärften Wettbewerb gewinnen kundenzentrierte Strategieansätze an Bedeutung. Für eine erfolgreiche Realisierung des Customer-Centricity-Ansatzes ist die Umsetzung durch die Mitarbeiter von hoher Relevanz (Schögel/Herhausen 2011). Denn im Dienstleistungssektor beeinflusst jeder Kontakt mit einem Mitarbeiter die Kundenwahrnehmung. In diesem Zusammenhang stellt sich die Frage, wie es gelingt, sämtliche Mitarbeiter im Unternehmen für die Customer-Centricity-Strategie zu gewinnen.

\section{„Um Customer Centricity im Unternehmen einzuführen, ist - neben der Verankerung dieses Ziels in der Unternehmensstrategie - vor allem die Umsetzung durch die Mitarbeiter relevant."}

\section{Neuausrichtung der Graubündner Kantonalbank}

Die Graubündner Kantonalbank (GKB) wurde 1870 gegründet und verfügt über eine erfolgreiche Tradition als Universalbank. Heute sind 1.000 Mitarbeiter bei der Marktführerin in Graubünden tätig, verteilt auf über 60 Standorte. Anfang des letzten Jahrzehnts zeigten Marktforschungsergebnisse, dass sich die Bank nach einer langen Phase des Wachstums in einer Stagnationsphase befand. Die Gründe dafür lagen zum einen im Umfeld (Rezession, erstarkte Konkurrenz), waren zum anderen aber hausgemacht: Eine ungeklärte strategische Stoßrichtung, über Jahrzehnte gewachsene Strukturen, schwerfällige Prozesse sowie nicht mehr zukunftsfähige, hochkomplexe IT-Systeme führten zu steigenden Kosten (bei stagnierenden Erträgen), basierend auf einer vom langjährigen Erfolg gesättigten Unternehmenskultur.

2002 überzeugte der frisch gewählte CEO die Entscheidungsträger der Bank, dass die Stagnationsphase - aus der bestehenden Position der Stärke heraus - durch eine konsequente Neuausrichtung der Bank auf die Kundenbedürfnisse zu überwinden sei. Das strategische Commitment bestand darin, dass die Kunden bei der GKB künftig kompetenter beraten werden und ein höheres Service-Level erfahren als bei der Konkurrenz. Diese Neupositionierung erforderte eine Reorganisation von einer "traditionellen, etwas schwerfälligen Universalbank“ hin zu einer „modernen, schlanken Vertriebsbank“, deren Kernkompetenz in der Beratung und Betreuung besteht. Im Folgenden wird erläutert, wie diese Customer-Centricity-Strategie rea-
Dr. Alex Villiger

ist Leiter Personal bei der Graubündner

Kantonalbank, Chur, Schweiz

E-Mail: alex.villiger@gkb.ch

Prof. Dr. Dennis Herhausen

ist Assistenzprofessor und Projektleiter am Institut für Marketing,

Universität St. Gallen (HSG), Schweiz

E-Mail: dennis.herhausen@unisg.ch

Prof. Dr. Marcus Schögel

ist Direktor am Institut für Marketing,

Universität St. Gallen (HSG), Schweiz

E-Mail:marcus.schögel@unisg.ch 
lisiert wurde und welche Wertschöpfung für die verschiedenen Anspruchsgruppen erzielt werden konnte.

\section{Phase 1: Kostenoptimierung und Gestaltung der harten Faktoren (ab 2002)}

In einem ersten Schritt wurde die Neuausrichtung der Bank in der Strategie und im Leitbild verankert - und damit die Grundlagen zu einer Neu-Positionierung als Vertriebsbank gelegt.

Im zweiten Schritt ist die GKB im Rahmen einer Gesamtbank-Reorganisation in die Struktur einer prozessorientierten Vertriebsbank übergegangen. Gleichzeitig hat sie sich einem Entschlackungs- und Kostensenkungsprogramm unterzogen und innerhalb eines Jahres sämtliche Kosten im Unternehmen um $5 \%$ gesenkt. Hieraus entstanden Freiräume, die Investitionen in die Kundenthemen ermöglichten. In der Folge konnten durch das Redesign der Kernprozesslandschaft Produktivitätssteigerungen erzielt werden, mit dem „positiven Nebeneffekt", dass die Bank ihren Kunden mit schlankeren, schnelleren und weniger fehleranfälligen Prozessen bessere Dienstleistungen anbieten konnte.

Das neue Geschäftsmodell (siehe Abbildung 1) verdeutlicht, dass bei der GKB alle Mitarbeiter die Wahrnehmung der Kunden positiv beeinflussen können: entweder direkt in der Kundenberatung („Frontoffice“) oder indirekt durch die Art und Weise, wie Dienstleistungen entwickelt und angeboten werden („Backoffice“).

\section{Abb. 1 Neues Geschäftsmodell der Graubündner Kantonalbank}

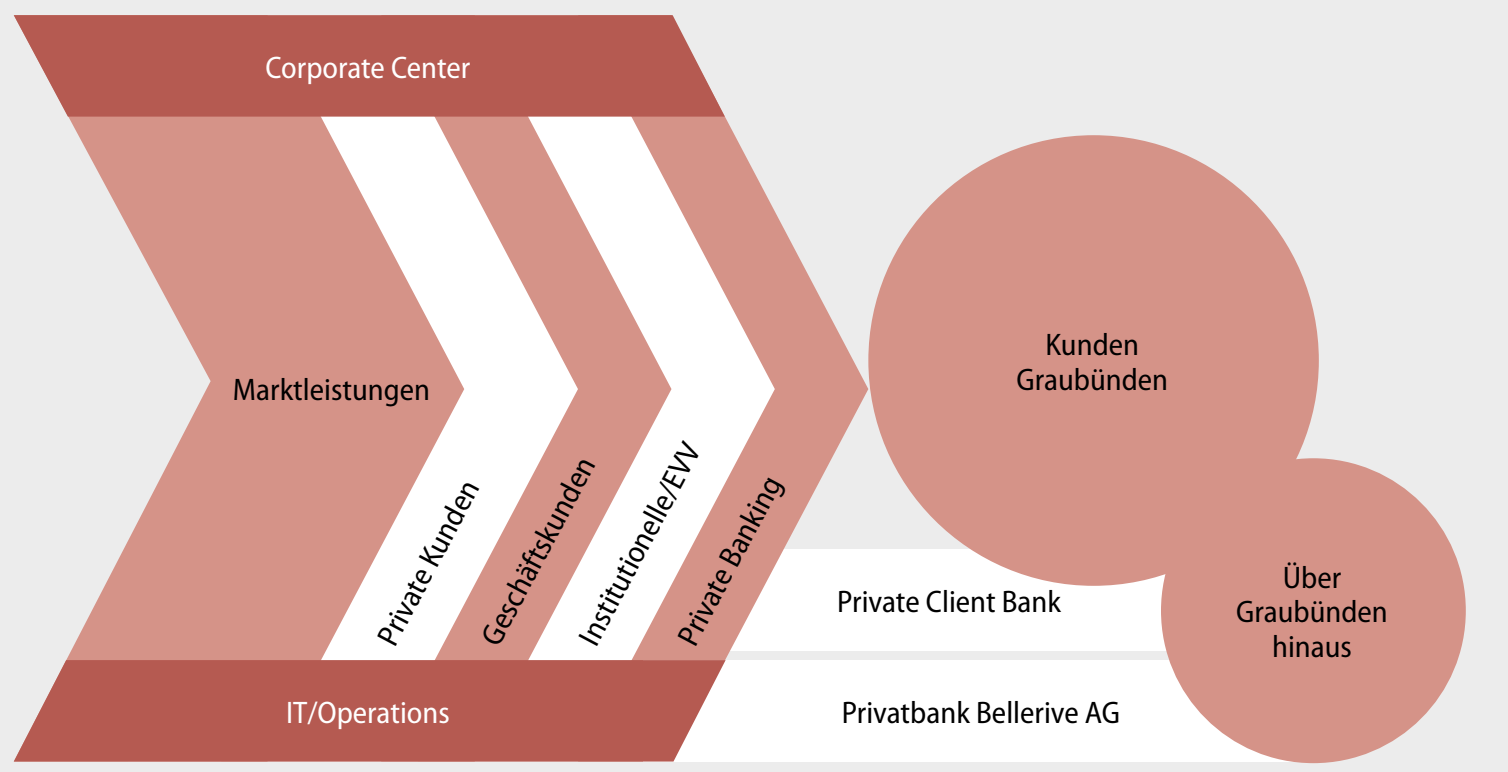

Quelle: Graubündner Kantonalbank (GKB) 
Nach der erfolgreichen Reorganisation wartete das nächste große Projekt. Die Geschäftsleitung gelangte 2004 zur Erkenntnis, dass Eigenentwicklung und Betrieb der Informatik zu viele Ressourcen banden. Zudem zeigte sich, dass ein Unternehmen in dieser Größe längerfristig nicht mehr über die finanziellen Ressourcen und Kompetenzen verfügt, um der steigenden Innovationsdynamik in der Systementwicklung gerecht zu werden. In der Folge lagerte die GKB ihre Informatik an eine Drittfirma aus, wagte gleichzeitig eine Migration auf eine standardisierte Bankenplattform und realisierte so weitere Kostensenkungspotenziale.

Parallel zu diesen Kostensenkungsmaßnahmen wurden in diesen ersten Jahren des Wandels Initiativen zur Optimierung der Kundenschnittstelle

\section{„Den Kunden gegenüber verspricht die Bank: 'gemeinsam wachsen. Dies erfordert eine hohe Beratungskompetenz wie auch einen hohen Service-Anspruch (Best Service-Ansatz).“}

realisiert. So hat die GKB im Jahr 2004 als erste Schweizer Bank einen ganzheitlichen Beratungsprozess eingeführt, der konsequent an den Kundenbedürfnissen ansetzt. Ein Jahr später wurde das strategische Geschäftsfeld Private Banking im Markt neu positioniert. Und 2006 hat die GKB am Hauptsitz in Chur ein modernes, offen gestaltetes und architektonisch prämiertes Kundenhaus eröffnet, welches Kunden und Mitarbeitern verdeutlichte, dass fortan die Interessen der Kunden am Ausgangspunkt des unternehmerischen Selbstverständnisses der Bank stehen.

\section{Konsequente Ausrichtung auf die Kundenschnittstelle}

Als Zwischenfazit dieser ersten Phase des Wandels kann festgehalten werden, dass es der Bank gelang, ihre Kosten in den Griff zu bekommen und die harten Unternehmensfaktoren auf die Kundenschnittstelle auszurichten. Die Kunden spürten jedoch noch wenig konkreten Nutzen. So gelangten die Promotoren des Wandels 2005 zur Erkenntnis, dass „die Kundenorientierung davon lebt, dass sie im gesamten Unternehmen auch wirklich gelebt wird“. Folglich galt es nun, das Verhalten der Mitarbeiter stärker auf die Kundenwünsche auszurichten: Die Kantonalbank stand vor einem Kulturwandel, welcher - im Gegensatz zum Kostenmanagement - nicht topdown angeordnet werden konnte. Erfolgskritisch für den Kulturwandel in Phase 2 war vielmehr, die Mitarbeiter für die Customer Centricity-Strategie zu gewinnen.

\section{Zusammenfassung}

Customer Centricity ist eine effektive Strategie, wenn es darum geht, Unternehmen aus einer Stagnationsphase herauszuholen und in eine anhaltende Wachstumsphase zu überführen.

Das Beispiel der Graubündner Kantonalbank (GKB) zeigt, wie durch einen langfristigen und ganzheitlichen Veränderungsprozess das gesamte Unternehmen auf die Kundenschnittstelle ausgerichtet werden kann.

Dieser Prozess führte nicht nur zu begeisterten Kunden, sondern hat darüber hinaus auch die Wertschöpfung der Bank nachhaltig erhöht. 


\section{Phase 2: Kulturwandel und Gestaltung der weichen Faktoren (ab 2006)}

Reichte es aus Sicht der Mitarbeiter bei der Ausübung ihrer Funktion bislang, sich auf ihre Bankexpertise abzustützen („Inside-Out-Perspektive“), galt es nun, diese Sicht um die Dimension der Kundenpräferenzen zu ergänzen („Outside-in-Perspektive“).

Die Suche nach Best-Practice-Ansätzen mit Ausrichtung auf die Kundenschnittstelle blieb erfolglos. So schuf die Unternehmensentwicklung der GKB ein eigenes Konzept zur Gestaltung der weichen Faktoren, das sich in seiner Logik am bekannten - und in Phase 1 realisierten - Modell zur Gestaltung der harten Faktoren anlehnte (siehe Abbildung 2). Analog zum „Framework zur Gestaltung der harten Faktoren“ („Strategie“) beinhaltet das „Framework zur Gestaltung der weichen Faktoren“ mit dem Thema „Markenführung“ ein Steuerungselement. Dieses verdeutlicht Kunden wie Mitarbeitern, wofür das Unternehmen steht und wie es sich im Markt zu positionieren gedenkt - das Thema „Markenführung“ übersetzt strategische Ziele in Verhaltensvorgaben (King/Grace 2012). Während die unternehmerische Leistungserstellung in Unternehmen - wie im ersten Modell dargelegt - durch die wechselseitige Wirkung von Aufbau- (Struktur) und Ablauforganisation (Prozesse) entsteht, entfaltet sich ein Kulturwandel im Dialog zwischen Führungskräften („Leadership“) und Mitarbeitern („Mitunternehmertum“). Schließlich sind es beim „Framework zur Gestaltung der harten Faktoren" Systeme und Instrumente, welche die Geschäftsabläufe tragen und deren Abbildung ermöglichen. Beim Framework zur Gestaltung der weichen Faktoren sind es HR-Kernprozesse und -Systeme, die den Kulturwandel unterstützen.

Im Folgenden wird beschrieben, wie die GKB das „Framework zur Gestaltung der weichen Faktoren“" zum Leben erweckt hat (Schritt 1 - 4).

\section{Abb. 2 Bezugsrahmen zur Realisierung der Customer Centricity}

Gestaltung der harten Faktoren

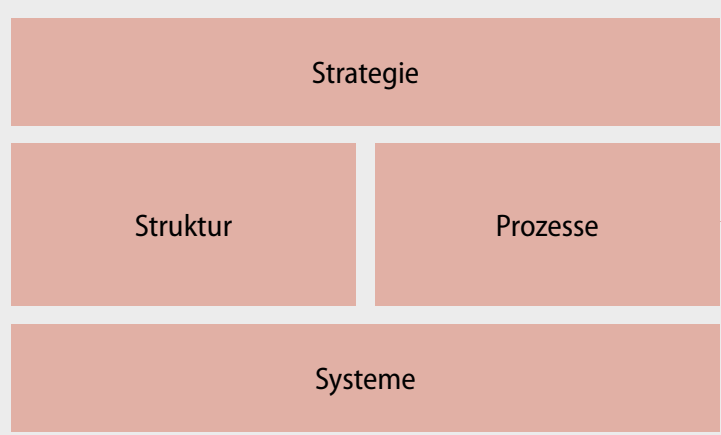

Gestaltung der weichen Faktoren

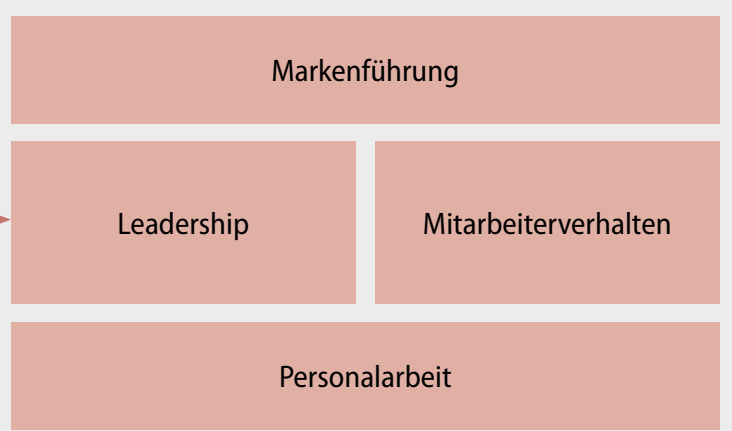

Quelle: Alex Villiger, Graubündner Kantonalbank (GKB) 


\section{Schritt 1: Versprechen abgeben - Markenführung gegen außen}

In Schritt 1 zur Gestaltung der weichen Faktoren wurde die Markenführung der Bank neu gestaltet: So wurden die bestehenden Fraktalmarken (jedes strategische Geschäftsfeld pflegte seine eigene Marke) zugunsten einer Dachmarkenstrategie aufgelöst. Dieser kommt die Aufgabe zu, den Anspruchsgruppen aufzuzeigen, welchen Nutzen sie von einer Zusammenarbeit mit der GKB erwarten dürfen. Die Markenverantwortlichen haben hierzu das Leitbild radikal vereinfacht. Dieses verdeutlicht, dass die Bank ihre Marktführerschaft dann ausbauen und die Stagnation hinter sich lassen dürfte, wenn sie Mitarbeiter mit einem persönlichen Führungsanspruch anspricht und entwickelt. Den Kunden gegenüber verspricht die Bank „gemeinsam wachsen“, was eine hohe Beratungskompetenz wie auch einen hohen Service-Anspruch beinhaltet. Schließlich galt es, das Leitbild mit Werten zu unterlegen: „Kompetenz" und „Verbundenheit“ ist dasjenige Wertepaar, das - in der Kombination gelebt - die GKB aus Kundensicht von der Konkurrenz abgrenzt.

Mit dem vom Kantonalbankenverband übernommenen Markenversprechen "gemeinsam wachsen“ signalisiert die Bank, dass die Kunden eine nachhaltige, wertschöpfende Beratung und Betreuung erwarten dürfen. Das Markenversprechen wurde schließlich im Rahmen aufwändiger Kampagnen im Markt verankert. Die mehrjährige Markeninszenierung beruhte auf der Darstellung von glücklichen Kunden, die mittels Bildsprache vermitteln, wie sie mit der GKB als Partner "gemeinsam wachsen“ - unabhängig davon, in welcher Lebensphase sich ein Unternehmen, eine Person oder eine Familie gerade befindet. Ein lückenloses Lebensphasenkonzept bildet das Fundament für die Zielgruppenansprache und für die Bündelung der zur Lebensphase passenden Produktangebote.

Wie neutrale Marktforschungsergebnisse belegen, erreichte die GKB in den Zielmärkten bereits nach wenigen Jahren in sämtlichen relevanten Dimensionen der Markenführung Spitzenwerte. Im Bewusstsein der Zielgruppen wurden die neuen Markeninhalte erfolgreich positioniert - und damit Erwartungen der Kunden geweckt.

\section{Schritt 2: Versprechen einlösen - Markenführung nach innen}

Parallel zur Verankerung in den Absatzmärkten hat die Brand Group der GKB den Mitarbeitern die Markenwerte nähergebracht (vgl. auch Tomczak et al. 2012). Hierzu wurden etwa folgende Initiativen lanciert: Kommunikation von erfolgreichen Kundenkontakten im Intranet („Storyboard“, Blog), konsequentes Re-Branding von Hilfsmitteln und Produkten (Schreibstifte, Ordner, Präsentationen, Schirme usw.), Produktion eines Unternehmensfilmes, Durchführung einer Mode-Show von Lernenden zur Lancierung einer eigenen Kleiderkollektion für die Freizeit, Verteilung von überraschenden Produkten mit Symbolgehalt (Tischpyramide mit Strategieinhalten, Bücher zur Reflexion der eigenen Change-Haltung,

\section{Kernthese 1}

Customer Centricity beauftragt alle Mitarbeiter, die Kundenbedürfnisse als Ausgangspunkt ihrer Arbeit zu interpretieren. Marketing wird zum Grundverständnis im Unternehmen, da sämtliche Funktionen einen Beitrag für das positive Kundenerlebnis leisten können - entweder in Form einer kompetenten Beratung im direkten Kundenkontakt oder durch das Erstellen kundengerechter Dienstleistungen im Backoffice. 


\section{Kernthese 2}

\section{Customer Centricity ist ein Veränderungsprozess, in des- sen Verlauf das gesamte Unter- nehmen auf die Kunden- schnittstelle ausgerichtet wird. Dabei gilt es in einer ersten Phase, die harten Faktoren zu gestalten (Rahmenbedingun- gen setzen), bevor in einer zweiten Phase die weichen Faktoren geprägt werden (Kul- turwandel).}

GKB-Pin etc.) oder die Übersetzung des Unternehmens- in Bereichsleitbilder durch die jeweiligen Teams. Schließlich hat die GKB neue Kommunikationsinstrumente lanciert, wie zum Beispiel den Welcome-Day zur Begrüßung der Neu-Eintretenden oder den CEO-Brunch, an dem Mitarbeiter in lockerer Atmosphäre Antworten zur Entwicklung der Bank erhalten.

Nebst diesen internen Branding-Maßnahmen hat die GKB 2008 die erste Wandelarena durchgeführt: In Großgruppenveranstaltungen hat sie den Mitarbeitern die Zusammenhänge zwischen dem Markenversprechen und ihrem Verhalten im Kundenkontakt nähergebracht. Den Teilnehmern wurde aufgezeigt, dass eine Erfolg versprechende Zukunft des eigenen Unternehmens davon abhängt, dass sämtliche Mitarbeiter die Markenwerte verinnerlichen und bei jedem Kundenkontakt leben. Indem gegenüber den Kunden zur Markenführung ein relevantes Versprechen abgegeben wird, entsteht eine Erwartungshaltung. Und wenn die Mitarbeiter dieses Markenversprechen täglich einlösen, entstehen Vertrauen und Kundenbegeisterung - als Basis für weiteres Wachstum bei bestehenden Kunden sowie bei der Erhöhung der Weiterempfehlungsbereitschaft zur Gewinnung neuer Kunden. Anschließend leiteten sämtliche Mitarbeiter für sich individuelle Ziele und Maßnahmen ab, wie sie die GKB auf ihrem Pfad der strategischen Zielerreichung noch wirkungsvoller unterstützen können. Dieser ganztägige Workshop löste nicht nur das erforderliche Change-Momentum aus, sondern stützte auch den Entwicklungsprozess bei jedem Mitarbeiter, die Markenbotschaften der Bank zu verinnerlichen. Dieser „Taking-in-Effekt" ermöglicht es wiederum, dass die Mitarbeiter künftig auch bei außerordentlichen Kundenanliegen in der Lage sind, das Markenversprechen auf natürliche und authentische Weise einzulösen. Die positive Gestaltung der „Moments of truth“ hilft, „Kunden zu Fans zu machen“ und die GKB gegenüber der Konkurrenz positiv abzugrenzen.

Die 2009 erstmals durchgeführte Kultur- und Führungsanalyse deckte auf, dass die Belegschaft der GKB zwar über gute, aber noch nicht über hervorragende Strategiekenntnisse verfügte. In der Folge wurde eine Wandelarena II angeboten, anlässlich derer die Geschäftsleitung den Mitarbeitern die Strategieinhalte erneut näher brachte.

\section{Schritt 3: Führung - Vom Management zum Leadership}

Den Verantwortlichen wurde in dieser Phase des Wandels bewusst, dass der Übergang zu echten, kundenorientierten Dienstleistern eine Veränderung im Führungsverhalten erforderte. Denn Kundenbegeisterung lösen nur Mitarbeiter aus, die auf ihren Arbeitgeber stolz sind, inspirierend geführt werden sowie ihren eigenen Beitrag zur Gesamtwirkung des Unternehmens kennen. In Zusammenarbeit mit den HSG-Professoren Marcus Schögel (Marketing) und Wolfgang Jenewein (Leadership) haben die Personalverantwortlichen ein Management-Development-Konzept entwickelt, das auf Marketing/Kundenorientierung sowie Führung/Leadership aufbaut: In den folgenden drei Jahren besuchten sämtliche Führungskräfte jährlich ein zweitägiges Marketing-Seminar. Dieses hatte zum Ziel, dem 
Kader das Thema Marketing/Kundenorientierung näherzubringen, sodass diese in der Neuausrichtung ihrer Teams mehr Sicherheit und Substanz erhielten.

In den nächsten drei Jahren folgte im Rahmen der Leadership-Seminare eine vertiefte Reflexion zum eigenen Führungsverhalten. Hier wurde das Ziel verfolgt, den erforderlichen Übergang von „weniger Management“ $\mathrm{zu}$ „mehr Leadership“ als vorherrschenden Führungsstil in der Bank zu etablieren.

Das Entwicklungskonzept dieser beiden Seminarreihen sah vor, dass sich stets 15 bis 20 Führungskräfte in unterschiedlichen Konstellationen aktuellen Marketing- und Führungsthemen widmeten. So konnte - parallel zum erforderlichen Wissenstransfer - die kollektive Intelligenz der Führungsebene für die Gestaltung der Unternehmensentwicklung genutzt werden. Zudem entstand eine enge Vernetzung aller Führungskräfte mit dem Vorteil, dass diese gegenseitiges Verständnis für die unterschiedlichen Realitäten in den Geschäftseinheiten aufbauen konnten. Wie sich später herausstellte, war Letzteres im Hinblick auf die gemeinsame Ausrichtung der operativen Einheiten auf die Kundenwünsche von zentraler Bedeutung.

„Management“ als Führungsstil der alten Welt kann so beschrieben wer-

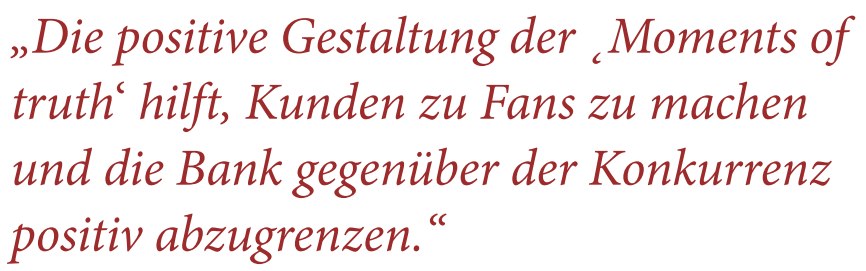

den, dass der Vorgesetzte - außerhalb des Teams stehend - Ziele (oft über die Köpfe des Personals hinweg) vorgibt. Nach einer Zeitperiode überprüft er, inwieweit seine Mitarbeiter die Ziele erreicht haben. Bei einer hohen Zielerreichung erhalten sie einen positiven Stimulus (Würdigung, Bonus sowie höhere Ziele für die nächste Periode). Im umgekehrten Fall ist mit Sanktionen zu rechnen (Rüge, tiefere Erfolgsbeteiligung, Entwicklungsmaßnahmen, ...). Die Mitarbeiter werden bei diesem sach-rationalen Führungsstil zu Ausführungskräften degradiert und verlieren oft ihre Freude und Leidenschaft sowie den Sinn ihrer Arbeit aus den Augen. Das vielgepriesene „Mitunternehmertum“ wird systematisch aus den Haltungen der Mitarbeiter verdrängt.

Eine Marketing-Organisation wie die GKB will Kunden aber über hohe Kompetenz und eine Best-Service-Kultur begeistern. Kreativität, intrinsische Motivation und Engagement sind folglich zentrale Faktoren, wenn es darum geht, über Beratung und Service Kundenbegeisterung zu wecken. Wie Professorin Morhart in ihrer Dissertation bei der GKB aufzeigte, kann die dafür erforderliche produktive Energie in der Belegschaft nur bei einem Führungsstil entstehen, der den Mitarbeitern das Gefühl von Autonomie (,ich wähle diese Einstellung aus freien Stücken“), Kompetenz („,ich

\section{Handlungsempfehlungen}

- Markenführung: Customer Centricity ist ein tauglicher Ansatz, um ein Unternehmen aus der Komfortzone zu locken und mit einer emotional aufgeladenen Vision auf einen gemeinsamen Kern auszurichten.

- Leadership: Customer Centricity wird nicht top-down angeordnet, sondern muss von den Mitarbeitenden selber gewählt und täglich gelebt werden. Dies erfordert einen Shift in der Führungsphilosophie eines Unternehmens im Sinne von „weniger Management und mehr Leadership“.

- Personalarbeit: Customer Centricity ist auf die zur Strategie passenden Menschen und Teams angewiesen. Eine clever inszenierte Arbeitgebermarke spricht geeignete Mitarbeitende im Arbeitsmarkt an. An den strategischen Vorgaben ausgerichtete Personalentwicklungskonzepte sorgen für den erforderlichen Entwicklungsschub im Unternehmen.

- Kostensenkung: Customer Centricity kann durchaus mit Kostensenkungsmaßnahmen gekoppelt werden („DualStrategie"), weil auch die Kunden von einer schlanken Organisation und schnellen, einfachen Prozessen profitieren.

- Ausdauer: Customer Centricity erfordert Ausdauer und nimmt etwa zehn Jahre in Anspruch. Diese lange Zeit ist der Grund, weshalb sich zwar viele Unternehmen Kundenorientierung auf die Fahne schreiben, aber im Verlauf der Realisierung kapitulieren. Kundenorientierung als Strategie lebt dann, wenn sämtliche Mitarbeiter sie täglich leben. 


\section{Kernthese 3}

Customer Centricity umzusetzen, ist ein komplexes Vorhaben, in dem verschiedene Fachstellen (Marketing, HRM, Unternehmensentwicklung) ihren Input leisten und in der Change-Dynamik ineinandergreifen müssen. Dies erfordert eine starke Führung und hohe Koordinationsfähigkeiten im Unternehmen. darf und kann auf die Kundenwünsche eingehen") und Verbundenheit zur Marke („ich will Teil dieser Bewegung sein“) vermittelt (Morhart 2008).

Die Führungsphilosophie des „Transformationalen Leadership“ wird diesen Anforderungen gerecht (Jenewein 2008) und grenzt sich damit deutlich vom transaktionalen Führungsstil („Management“) ab. Basis bilden die vier „i-Säulen“ (siehe Abbildung 3).

\section{Schritt 4: HRM - Personalarbeit auf Strategie ausrichten und Kulturwandel tragen}

Als letztes Element zur Realisierung des Kulturwandels wurde 2009 die Neugestaltung der Personalarbeit erkannt. Wie Studien belegen, hat die Qualität der Personalarbeit eine große Wirkung auf die Unternehmensresultate. Zentrale Themen bei der Neuausrichtung der Personalarbeit auf die Strategievorgaben der Bank waren einerseits der Aufbau einer starken Arbeitgebermarke, andererseits die Entwicklung strategischer Personalentwicklungskonzepte. Ziel der Personalmarketing-Initiative war es, im Arbeitsmarkt zum Unternehmen und zur Neuausrichtung der GKB passende Mitarbeiter anzusprechen, zu gewinnen und zu binden. Hierfür wurden folgende Maßnahmen realisiert:

- Erarbeitung einer Arbeitgeberpositionierung (siehe Abbildung 4),

- Entwicklung einer Arbeitgeberbroschüre,

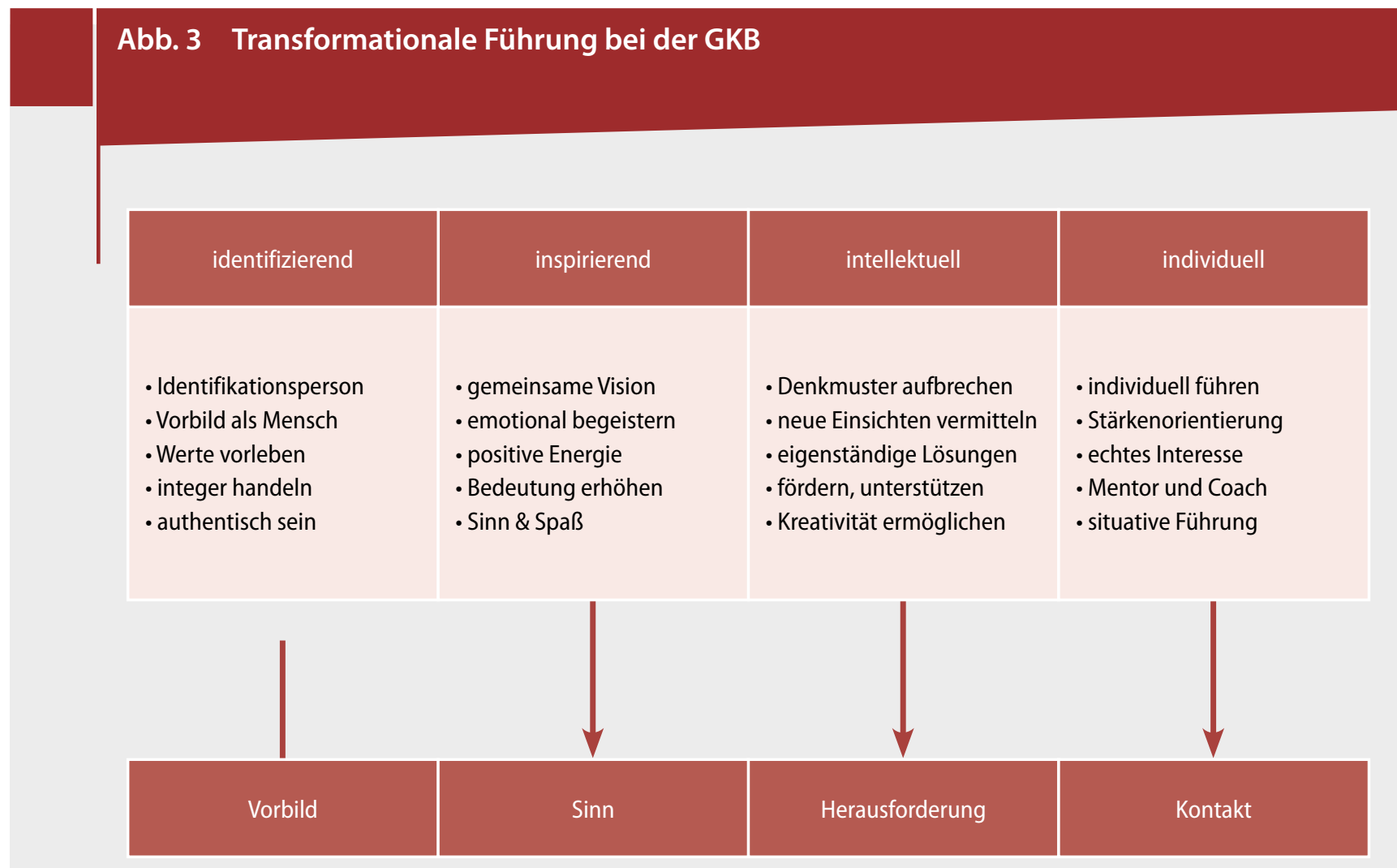


- Aufbau eines modernen Jobportals (htp://www.gkb.ch/jobs) samt elektronischem Rekrutierungssystem,

- Durchführung von Workshops mit Studienabgängern,

- Auftritte als Dozenten bei (Fach-)Hochschulen oder

- Publikationen in der Lokalpresse zu aktuellen HR-Fragen.

Zur Erfassung der Potenziale der bestehenden Mannschaft sowie zur Weiterentwicklung von deren Kompetenzen wurden ebenfalls Initiativen realisiert: so zum Beispiel die Abbildung der Unternehmensstrategie in einem Kompetenzmodell, individuelle Schulungskonzepte für größere Bereiche, Entwicklungsmodule für Kundenberater, Durchführung von Stage-Programmen oder die Einführung eines Talent-Management-Konzepts.

Im Rahmen eines Reorganisationsprogramms hat die Bank sämtliche HRProzesse konsequent auf die Unternehmensstrategie ausgerichtet und sich für die Begleitung des Kulturwandels in Richtung moderne, erfolgreiche Vertriebsbank gerüstet. Die Arbeitgeberpositionierung dient dabei der Neuausrichtung der HR-Kernprozesse als Orientierungsgröße (im inneren Kreis das Leitbild mit den Anforderungen an künftige Arbeitskräfte; im äußeren Kreis die drei Arbeitgeberwerte, die interessierte Mitarbeiter von der GKB als Arbeitgeberin erwarten dürfen).

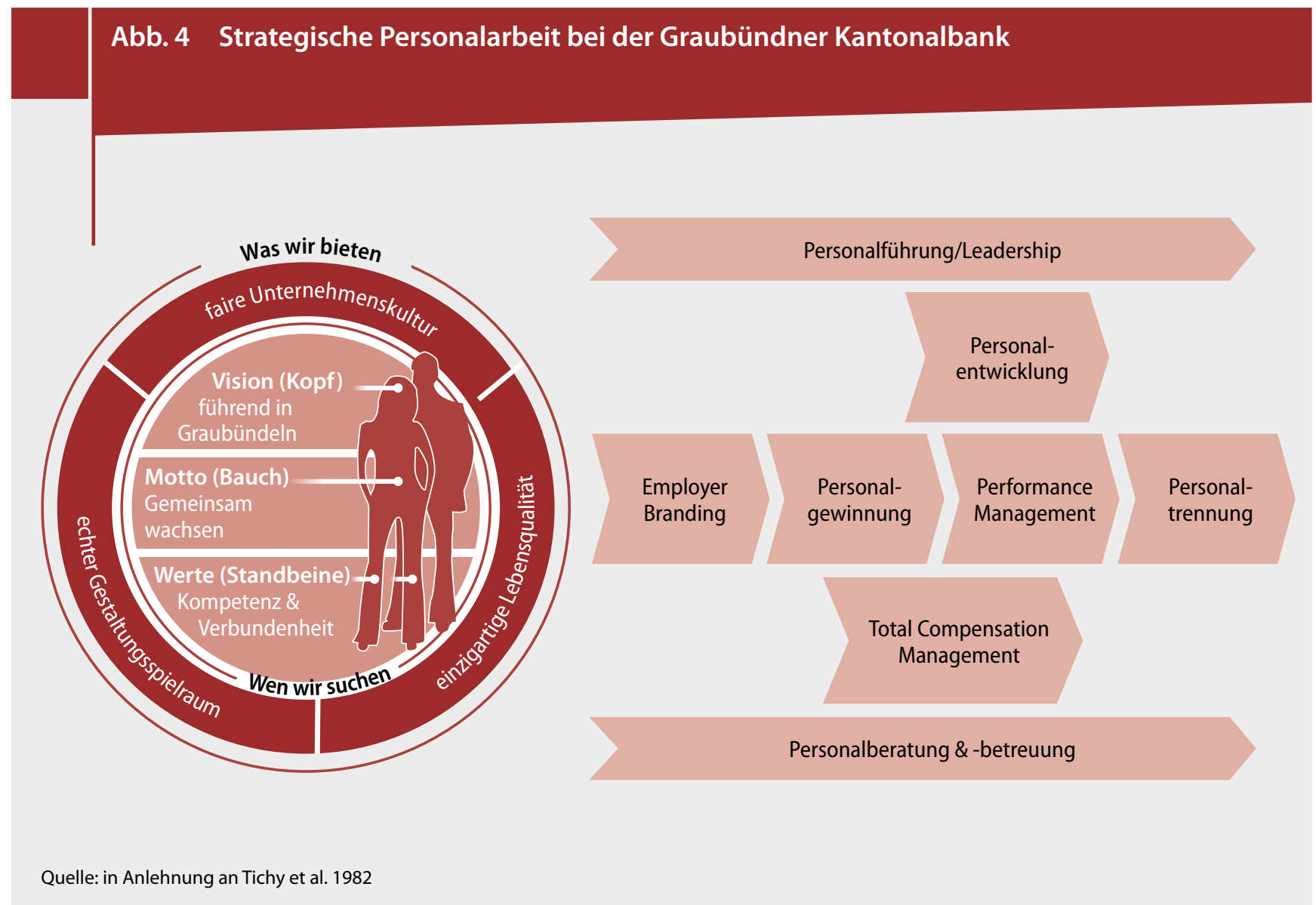




\section{Fazit und Wirkung}

Will ein Unternehmen in einem umkämpften Markt erfolgreich sein, ist es auf begeisterte Kunden angewiesen, die zudem bereit sind, ihren bevorzugten Partner weiterzuempfehlen. Basis hierfür sind begeisterte Mitarbeiter, die auf ihren Arbeitgeber stolz sind und diese Haltung im Kundenkontakt übertragen. Wenn es um die Ergründung und Befriedigung von Kundenwünschen geht, sind ein hohes Commitment sowie ein hohes Level an produktiver Energie Voraussetzung. Mitarbeiter sind in der Regel dann bereit, für Kundenbelange die berühmte Extra-Meile zu gehen, wenn die Arbeitsbedingungen stimmen, Entwicklungsperspektiven und Gestaltungsspielräume gegeben sind, die Unternehmenskultur als fair empfunden wird und wenn sie gut geführt werden. Mitarbeitergerechte Führung orientiert sich im modernen Dienstleistungsunternehmen an den Kriterien des transformationalen Leaderships. Wertschöpfung und Unternehmenserfolg beginnen folglich mit Investitionen in die Führungsqualität. So zeigte eine groß angelegte Studie von Morhart et al. (2012), dass sich der Umgang der Vorgesetzten mit den Mitarbeitern unmittelbar auf deren Beziehung zu den Kunden auswirkt. Wenn eine Führungskraft die geforderten Werte vorlebt, wirkt sie glaubhaft und kann zur „Nachahmung“ motivieren. Führungskräfte vermitteln Sinn und Bedeutung der Customer Centricity durch ihre wertorientierte Führung. Nur so können sie begeistern, als Vorbilder wahrgenommen werden und zur Wertschöpfung des Unternehmens beitragen.

\section{Abb. 5 Die Wirkungskette der Customer Centricity}

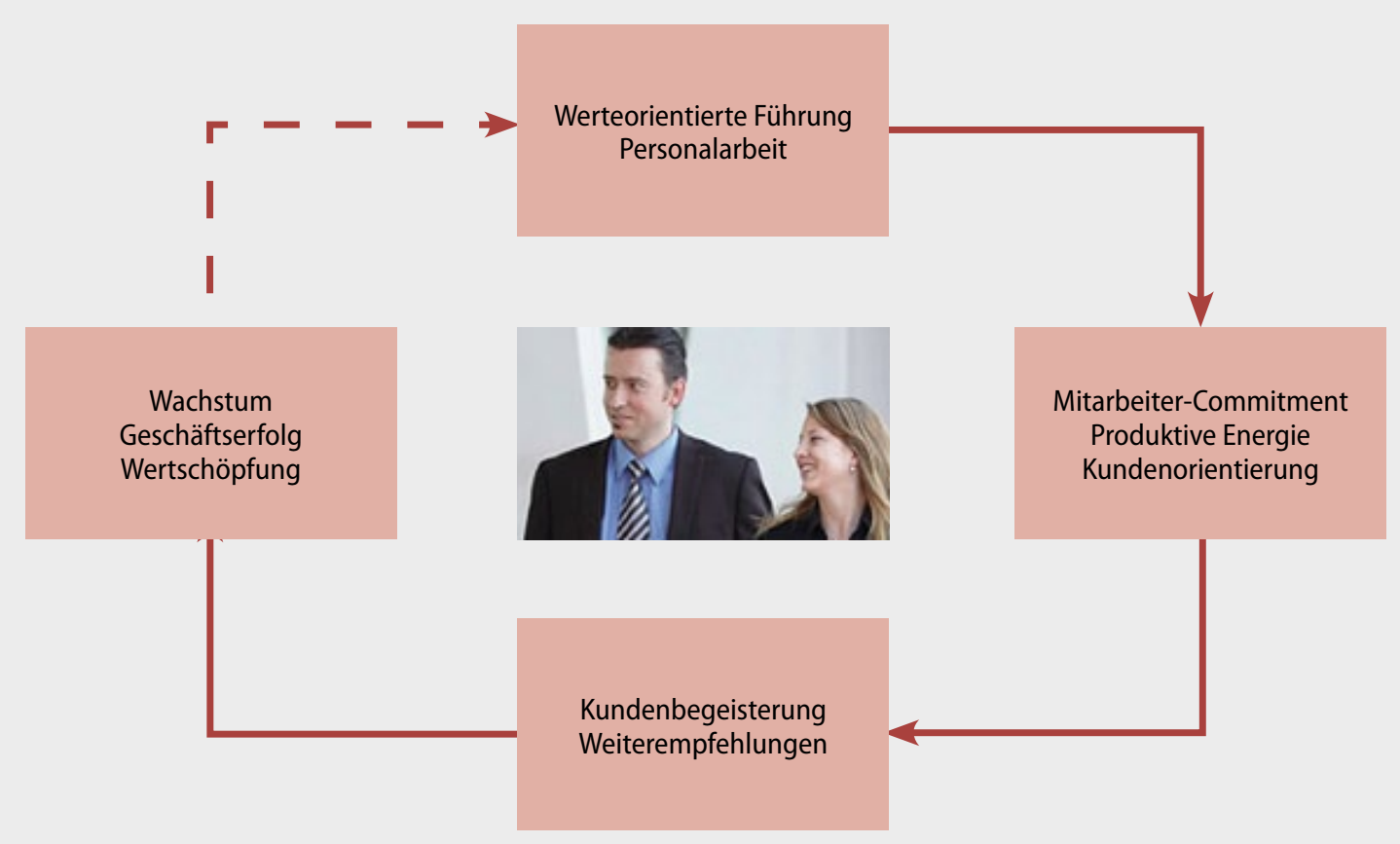

Quelle: Graubündner Kantonalbank (GKB) 
Der in Abbildung 5 dargestellte Kreislauf wurde bei der GKB zuerst durch die Analyse verschiedener Kennzahlen in Controlling- und Marktforschungsreports begründet. In einem zweiten Schritt vertieft und quantifiziert die Bank derzeit mit dem Institut für Marketing der Universität sowie

der energy factory St. Gallen die Wirkung der dargestellten Treiber zur Umsetzung der Customer-Centricity-Strategie. So konnte in einem ersten Schritt durch ein Strukturgleichungsmodell nachgewiesen werden, dass eine ausgeprägte transformationale Führung mit einem Pfadkoeffizienten in Höhe von 0.40 auf die produktive Energie der Mitarbeiter, und diese wiederum mit 0.36 auf die Kundenorientierung wirkt.

\section{Erfolge der Customer-Centricity-Strategie}

Dank ihrer strategischen Neuausrichtung konnte sich die GKB bei allen relevanten Kennzahlen signifikant verbessern. Folgende Indikatoren belegen den Erfolg der Customer-Centricity-Strategie:

- Investoren: Analog zum Walker-Index (Walker 2013) in den USA erzielte der GKB-Titel im beschriebenen Zeitraum (2002-2012) eine Outperformance von über 100 Prozentpunkten gegenüber den gängigsten Indizes an der Schweizer Börse (SPI, SMI).

- Analysten: Die gewählte Strategie der Kundenausrichtung, die dadurch generierte Wertschöpfung, die Wachstumsaussichten, die Produktivität der Bank und andere Kennzahlen veranlassten Vontobel im Jahr 2009, die GKB als einzige Kantonalbank mit dem Stand-alone-Rating AA- auszuzeichnen. Standard \& Poor's vergibt der GKB regelmäßig das zweithöchste Rating AA+.

- Kunden: Die Kundenzufriedenheit ermittelt die GKB durch Marktforschungsumfragen. Dabei zeigt sich, dass die Zufriedenheit der Kunden mit einer Hauptbankbeziehung zur GKB seit 2003 kontinuierlich gestiegen ist. Auch die Empfehlungsbereitschaft hat stark zugenommen. Seit 2007 definiert die GKB zudem einen neuen Kundentyp - den „Apostel“. Dieser empfiehlt die GKB weiter und stehen auch emotional hinter ihr. Der Anteil der Apostel liegt heute bei ca. 70 \% der Kunden.

- Management: Während die Erträge seit 2003 konsequent steigen, konnten die Kosten dank der gewählten Dual-Strategie (Kundenausrichtung bei gleichzeitigem Kostenmanagement) konstant gehalten werden. Heute bewältigt die Bank im Vergleich zum Start ihrer Neuausrichtung ein um

\section{Kernthese 4}

Customer Centricity gleicht einer Vision, die man als Unternehmen nie ganz erreichen wird, der man sich durch die Lancierung geeigneter Initiativen jedoch annähern kann. 
über 50 \% höheres Geschäftsvolumen - mit derselben Anzahl von Mitarbeitern wie vor zehn Jahren!

- Mitarbeiter: Auf der unabhängigen Plattform www.kununu.com können Mitarbeiter ihre Arbeitgeber anonym beurteilen. Die GKB rangiert wiederholt in den Top 3 sämtlicher Schweizer Unternehmen sowie an erster Stelle in der Kategorie Schweizer Banken. Das international renommierte CRF-Institute vergibt der GKB seit 2011 jährlich das Label „Top Arbeitgeber".

- Interne Umfragen zur Kultur und Führung der Bank weisen Teilnahmequoten von gegen $90 \%$ auf. Davon empfehlen wiederum beinahe 90 Prozent die GKB als Arbeitgeberin wie auch als Anbieterin von Bankdienstleistungen uneingeschränkt weiter. Die produktive Energie im Unternehmen, die von der energy factory in St. Gallen alle drei Jahre erhoben wird, steigt kontinuierlich an und weist heute einen Wert von deutlich über $70 \%$ aus. Das bringt die GKB in den Bereich der besten Unternehmen in dieser Benchmark-Erhebung (Bruch/Kunz 2009).

Insgesamt zeigen diese Zahlen, dass alle Anspruchsgruppen von dieser Ausrichtung auf die Kundenschnittstelle profitieren. Sämtliche Umfragen und Studien weisen Wachstumszahlen auf. So auch die unten stehende Darstellung der Entwicklung der Bilanzsumme. Diese zeigt, dass die Stagnationsphase der Jahre 2000 bis 2002 dank konsequenter Umsetzung der CustomerCentricity-Strategie überwunden wurde und in einem Wachstum mündete, welches sich Jahr für Jahr deutlich über dem Markt bewegt.

\section{Abb. 6 Marktwachstum der Graubündner Kantonalbank}

Bilanzsumme GKB (in Milliarden Schweizer Franken):

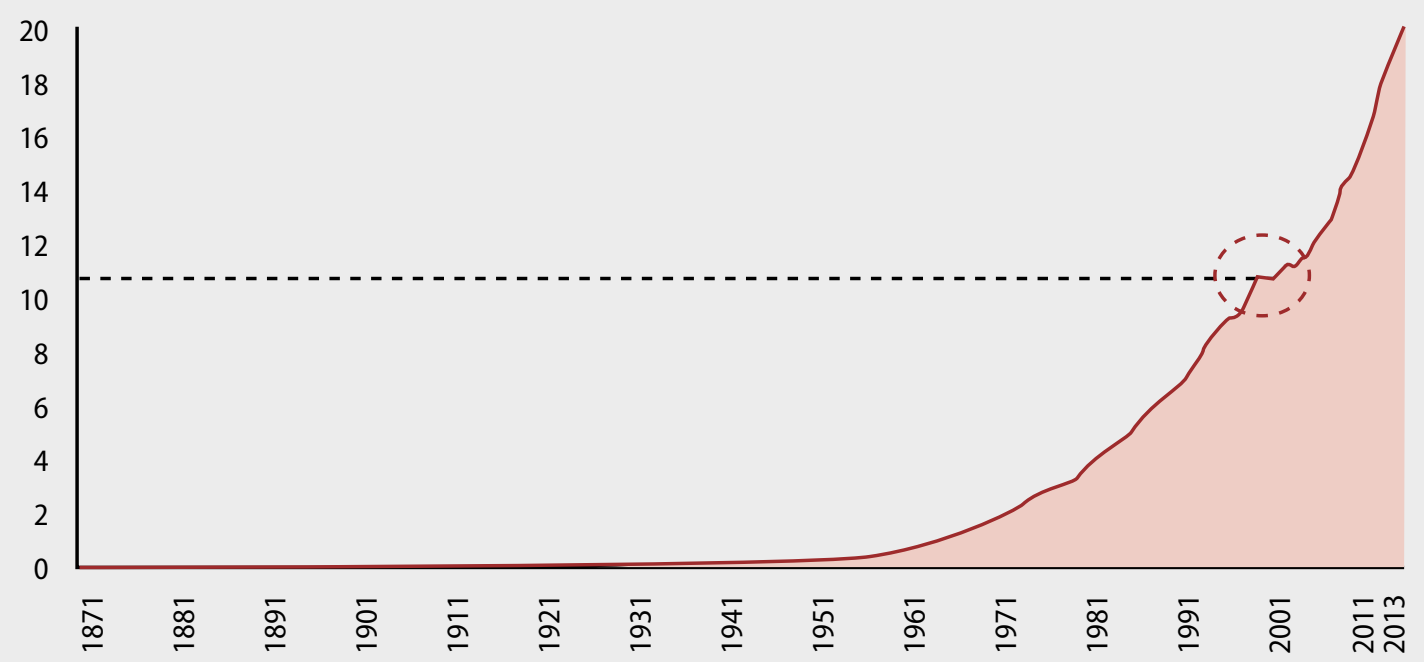




\section{Literatur}

Bass, B. M./Avolio, B. J. (1994): Improving Organizational Effectiveness Through Transformational Leadership, Thousand Oaks.

Bruch, H./Kunz, J. J. (2009): Organisationale Energie durch Personalarbeit freisetzen und erhalten, in: 2009 - Jahrbuch Personalentwicklung, S. 5-13.

Graubündner Kantonalbank (2013): Führungs- und Personalarbeitsverständnis der GKB (http://www.gkb.ch/jobs, letzter Abruf: 09.08.2013).

Jenewein, W. (2008): Change Management: Das Klinsmann Projekt, in: Harvard Business Manager 29, 6, S. 16-30.

King, C./Grace, D. (2012): Examining the Antecedents of Positive Employee BrandRelated Attitudes and Behaviours, in: European Journal of Marketing 46, 3, pp. 469-488.

kununu.com (2013): Graubündner Kantonalbank in Chur - Erfahrungsberichte, Bewertungen und Kommentare (http://www.kununu.com/ch/all/ch/bf/graubuendnerkantonalbank, letzter Abruf: 09.08.2013).

Morhart, F./Jenewein, W./Herzog, W./Brösamle, S. (2012): Guter Chef, Gute Verkäufer, in: Harvard Business Manager, 9, S. 44-46.

Morhart, F. (2008): Brand Specific Leadership: On ist Effect and Trainability. Dissertation, Universität St. Gallen.

Schögel, M./Herhausen, D. (2011): Stolpersteine auf dem Weg zum kundenzentrierten Unternehmen, in: Marke41, 4, S. 16-21.

Tichy, N. M./Fombrun, CH. J./Devanna, M.A. (1982): Strategic Human Resource Management, in: Sloan Management Review, 2, pp. 47-61.

Tomczak, T./Esch, F.-R./Kernstock, J./Herrmann, A. (2012): Behavioral Branding: Wie Mitarbeiterverhalten die Marke stärkt, 3. Aufl., Wiesbaden.

Walker (2013): Der Walker Index (http://www.walkerinfo.com, letzter Abruf: 09.08.2013).

Wunderer, R. (2009): Führung und Zusammenarbeit - eine unternehmerische Führungslehre. 9. Aufl., Köln.

Zusatzservice für Abonnenten von „Springer für Professionals | Marketing“

Zum Thema Customer Centricity

finden Sie unter www.springerprofessional.de 239 Beiträge, davon 46 im Fachgebiet Marketing.

\section{Medium}

Zeitschriftenartikel (8)

Buchkapitel (38)

\section{Sprache}

Deutsch (19)

Englisch (27)

\section{Von der Redaktion empfohlen}

Vogelaar, R. (2011): Die Evolution der Kundenorientierung, in: Der Superpromoter, Umsatz und Reputation steigern mit begeisterten Kunden, Wiesbaden, S. 63-96.

www.springerprofessional.de/1816534 